\title{
ON WEIGHTED MULTIDIMENSIONAL EMBEDDINGS FOR MONOTONE FUNCTIONS
}

\author{
SORINA BARZA, LARS-ERIK PERSSON and VLADIMIR D. STEPANOV*
}

\section{Abstract}

We characterize the inequality

$$
\left(\int_{\mathrm{R}_{+}^{N}} f^{q} u\right)^{1 / q} \leq C\left(\int_{\mathrm{R}_{+}^{N}} f^{p} v\right)^{1 / p}, \quad 0<q, p<\infty,
$$

for monotone functions $f \geq 0$ and nonnegative weights $u$ and $v$. The case $q<p$ is new and the case $0<p \leq q<\infty$ is extended to a modular inequality with $\mathrm{N}$-functions. A remarkable fact concerning the calculation of $C$ is pointed out.

\section{Introduction}

Let $\mathrm{R}_{+}^{N}:=\left\{\left(x_{1}, \ldots, x_{N}\right) ; x_{i} \geq 0, i=1,2, \ldots, N\right\}$ and $\mathrm{R}_{+}:=\mathrm{R}_{+}^{1}$. Assume that $f: \mathrm{R}_{+}^{N} \rightarrow \mathrm{R}_{+}$is monotone which means that it is monotone with respect to each variable. We denote $f \downarrow$, when $f$ is decreasing (=nonincreasing) and $f \uparrow$ when $f$ is increasing (=nondecreasing).

Given $0<p, q<\infty$ and the weights $u \geq 0$ and $v \geq 0$ we consider the inequality

$$
\left(\int_{\mathrm{R}_{+}^{N}} f^{q} u\right)^{1 / q} \leq C\left(\int_{\mathrm{R}_{+}^{N}} f^{p} v\right)^{1 / p}
$$

for all $f \downarrow$ or $f \uparrow$.

In the one dimensional case the inequality (1) was characterized in ([4], Proposition 1) for both alternative cases $0<p \leq q<\infty$ and $0<q<p<\infty$ as follows:

(a) If $N=1,0<p \leq q<\infty$, then (1) is valid for all $f \downarrow$ if and only if

$$
A_{0}:=\sup _{t>0}\left(\int_{0}^{t} u\right)^{1 / q}\left(\int_{0}^{t} v\right)^{-1 / p}<\infty
$$

and the constant $C=A_{0}$ is sharp.

\footnotetext{
* Partially supported by the Swedish Institute grant $97-1331$, by the INTAS project $94-881$ and by the RFBR grant 97-01-00604.

Received May 12, 1998
} 
(b) If $N=1,0<q<p<\infty, 1 / r=1 / q-1 / p$, then (1) is true for all $f \downarrow$ if and only if

$$
B_{0}:=\left(\int_{0}^{\infty}\left(\int_{0}^{t} u\right)^{r / p}\left(\int_{0}^{t} v\right)^{-r / p} u(t) d t\right)^{1 / r}<\infty
$$

Moreover,

$$
\left(\frac{q^{2}}{p r}\right)^{1 / p} B_{0} \leq C \leq\left(\frac{r}{q}\right)^{1 / r} B_{0}
$$

and

$$
B_{0}^{r}=\frac{q}{r} \frac{\left(\int_{0}^{\infty} u\right)^{r / q}}{\left(\int_{0}^{\infty} v\right)^{r / p}}+\frac{q}{p} \int_{0}^{\infty}\left(\int_{0}^{t} u\right)^{r / q}\left(\int_{0}^{t} v\right)^{-r / q} v(t) d t
$$

(c) The same characterizations are valid, when $f \uparrow$, with the only replacement of the integrals over $[0, t]$ by the integrals over $[t, \infty]$.

Since the one dimensional inequality (1) expresses the embedding of classical Lorentz spaces, the further generalizations and references in this directions can be found in [2]. The multidimensional case was treated in ([1], Theorem 2.2), where, in particular the inequality (1) was characterized in the case $0<p \leq q<\infty$ and the sharp value of the constant $C$ was given as

$$
C=A_{N}:=\sup _{D \in \mathscr{D}_{d}} \frac{\left(\int_{D} u\right)^{1 / q}}{\left(\int_{D} v\right)^{1 / p}}
$$

and supremum is taken over the set $\mathscr{D}_{d}$ of all "decreasing" domains. Moreover it was shown ([1], Theorem 2.5) that if $u(x)$ and $v(x)$ are product weights, i.e., if

$$
u(x)=u_{1}\left(x_{1}\right) \ldots u_{N}\left(x_{N}\right), \quad v(x)=v_{1}\left(x_{1}\right) \ldots v_{N}\left(x_{N}\right),
$$

then the constant $C$ can be calculated in the following way:

$$
C=A_{N}^{(1)}:=\sup _{a_{i}>0} \frac{\left(\int_{0}^{a_{1}} \cdots \int_{0}^{a_{N}} u\right)^{1 / q}}{\left(\int_{0}^{a_{1}} \cdots \int_{0}^{a_{N}} v\right)^{1 / p}}
$$

It was also pointed out in [1], Example 3.1, that if $u(x)$ and $v(x)$ are not product weights, then the equality $A_{N}=A_{N}^{(1)}$ is not true in general. In fact, in this paper we even prove the remarkable fact that theconstants $A_{N}$ and $A_{N}^{(1)}$ are not comparable in general (for $N \geq 2$ ). 
Section 2 of the present paper is devoted to the modular inequality of the form

(8) $\Phi_{2}^{-1}\left(\int_{\mathrm{R}_{+}^{N}} \Phi_{2}(\omega(x) f(x)) u(x) d x\right) \leq \Phi_{1}^{-1}\left(\int_{\mathrm{R}_{+}^{N}} \Phi_{1}(C f(x)) v(x) d x\right)$,

where $\Phi_{1}$ and $\Phi_{2}$ are N-functions [3] such that

$$
\sum_{n} \Phi_{2} \circ \Phi_{1}^{-1}\left(a_{n}\right) \leq K \Phi_{2} \circ \Phi_{1}^{-1}\left(\sum_{n} a_{n}\right)
$$

for all $a_{n} \geq 0$ with a constant $K \geq 1$ independent on $\left\{a_{n}\right\}$.

In Section 3 we consider the particular case of (1), when $N=2,0<p \leq$ $q<\infty, u(x, y)=u(x y), v(x, y)=v(x y)$ and find an explicit criterion for this case. One important consequence of this result is that there is no uniform constant $c>0$ such that $c A_{N}^{(1)} \geq A_{N}$, i.e., $A_{N}$ and $A_{N}^{(1)}$ are not comparable in general.

The case $0<q<p<\infty$ of (1) is characterized in Section 4 .

Conventions and notations. Products and quotients of the forms $0 \cdot \infty$, $\frac{\infty}{\infty}, \frac{0}{0}$ are taken to be $0 . Z$ stands for the set of all integers and $\chi_{E}$ denotes the characteristic function of a set $E$.

ACKNOWLEDGMENT. The third named author wishes to thank all colleagues at the department of Mathematics at Luleå University of Technology for hospitality and interesting discussions during the research stay March-April 1998.

\section{A modular integral inequality}

Let $0 \leq h(x) \downarrow$ and $t>0$. Denote

$$
D_{h, t}:=\left\{x \in \mathrm{R}_{+}^{N} ; h(x)>t\right\},
$$

and

$$
\mathscr{D}_{d}:=\bigcup_{0 \leq h \downarrow} \bigcup_{t>0} D_{h, t}
$$

The set $\mathscr{D}_{d}$ consists of all "decreasing" domains $D_{h, t}$. In particular, $\chi_{D_{h, t}}$ is decreasing in each variable.

Let $\Phi: R_{+} \rightarrow R_{+}$be a nonnegative, convex function such that

$$
\lim _{x \rightarrow 0} \frac{\Phi(x)}{x}=0, \quad \lim _{x \rightarrow \infty} \frac{\Phi(x)}{x}=\infty .
$$


Following [3] we call $\Phi$ an N-function. In particular,

$$
\Phi(x) \leq \frac{1}{a} \Phi(a x) \quad \text { for all } \quad a \geq 1, \quad x>0 .
$$

THEOREM 2.1. Let $\Phi_{1}, \Phi_{2}$ be two $N$-functions satisfying (9). Given weight functions $\omega(x) \geq 0, u(x) \geq 0, v(x) \geq 0$ the inequality (8) holds for all $0 \leq f \downarrow$ if and only if there exists a constant $A=A\left(\Phi_{1}, \Phi_{2}, u, v, \omega\right)$ such that, for all $\varepsilon>0$ and $D_{h, t} \in \mathscr{D}_{d}$

$$
\Phi_{2}^{-1}\left(\int_{D_{h, t}} \Phi_{2}(\varepsilon \omega(x)) u(x) d x\right) \leq \Phi_{1}^{-1}\left(\Phi_{1}(A \varepsilon) \int_{D_{h, t}} v(x) d x\right) .
$$

Proof. The necessity follows, if we replace $f$ in (8) by $f=\varepsilon \chi_{D_{h, t}}$. For sufficiency we define for a fixed $f \downarrow$

$$
\begin{aligned}
& \Delta_{n}:=\left\{x \in \mathrm{R}_{+}^{N} ; 2^{n}<f(x) \leq 2^{n+1}\right\}, \quad n \in \mathrm{Z}, \\
& D_{n}:=\left\{x \in \mathrm{R}_{+}^{N} ; f(x)>2^{n}\right\},
\end{aligned}
$$

and note that

$$
D_{n} \supset D_{n+1}, D_{n}=\bigcup_{k \geq n} \Delta_{k}, \quad \mathrm{R}_{+}^{N}=\bigcup_{n} \Delta_{n} .
$$

Obviously, $\Delta_{n} \bigcap \Delta_{k}=\emptyset$ for $n \neq k$. We have, using (10)

$$
\begin{aligned}
\int_{\mathrm{R}_{+}^{N}} \Phi_{2}(\omega(x) f(x)) u(x) d x & \leq \frac{1}{K} \int_{\mathrm{R}_{+}^{N}} \Phi_{2}(K \omega(x) f(x)) u(x) d x \\
& =\frac{1}{K} \sum_{n} \int_{\Delta_{n}} \Phi_{2}(K \omega(x) f(x)) u(x) d x \\
& \leq \frac{1}{K} \sum_{n} \int_{\Delta_{n}} \Phi_{2}\left(2^{n+1} K \omega(x)\right) u(x) d x \\
& \leq \frac{1}{K} \sum_{n} \int_{D_{n}} \Phi_{2}\left(2^{n+1} K \omega(x)\right) u(x) d x
\end{aligned}
$$

[applying (11) with $\varepsilon=2^{n+1} K$ ]

$$
\leq \frac{1}{K} \sum_{n} \Phi_{2} \circ \Phi_{1}^{-1}\left(\Phi_{1}\left(A K 2^{n+1}\right) \int_{D_{n}} v\right)
$$


[applying (9)]

$$
\begin{aligned}
& \leq \Phi_{2} \circ \Phi_{1}^{-1}\left(\sum_{n} \Phi_{1}\left(A K 2^{n+1}\right) \sum_{k \geq n} \int_{\Delta_{k}} v\right) \\
& =\Phi_{2} \circ \Phi_{1}^{-1}\left(\sum_{k}\left(\int_{\Delta_{k}} v\right) \sum_{n \leq k} \Phi_{1}\left(A K 2^{n+1}\right)\right)
\end{aligned}
$$

[using the convexity of $\Phi_{1}$ ]

$$
\begin{aligned}
& \leq \Phi_{2} \circ \Phi_{1}^{-1}\left(\sum_{k}\left(\int_{\Delta_{k}} v\right) \Phi_{1}\left(4 A K 2^{k}\right)\right) \\
& \leq \Phi_{2} \circ \Phi_{1}^{-1}\left(\sum_{k} \int_{\Delta_{k}} \Phi_{1}(4 A K f(x)) v(x) d x\right) \\
& =\Phi_{2} \circ \Phi_{1}^{-1}\left(\int_{R_{+}^{N}} \Phi_{1}(4 A K f(x)) v(x) d x\right) .
\end{aligned}
$$

Thus, the least possible constant $C$ in (8) satisfies

$$
A \leq C \leq 4 A K \text {. }
$$

Theorem 2.1 is proved.

\section{Explicit criteria for some cases}

As we mentioned in the Introduction in the case of product weights (see (6)) the least possible constant $C$ in (1) satisfies (7). The natural and important question is whether the constants $A_{N}(5)$ and $A_{N}^{(1)}$ (7) are comparable in the general case. Clearly, $A_{N}^{(1)} \leq A_{N}$, but the converse inequality $A_{N} \leq c A_{N}^{(1)}$ with a constant $c$ independent on weights was so far uncertain. Below we give a negative answer to this question with the help of the following result:

Theorem 3.1. Let $0<p \leq q<\infty$ and $u(s) \geq 0, v(s) \geq 0$ be two measurable functions on $\mathrm{R}_{+}$such that $U(t):=\int_{0}^{t} u<\infty, V(t):=\int_{0}^{t} v<\infty$ for all $t>0$.

Then the inequality

$$
\left(\int_{\mathrm{R}_{+}^{2}} f^{q}(x, y) u(x y) d x d y\right)^{1 / q} \leq C\left(\int_{\mathrm{R}_{+}^{2}} f^{p}(x, y) v(x y) d x d y\right)^{1 / p}
$$

holds for all $f(x, y) \geq 0$ decreasing in $x$ and $y$ with a finite constant $C>0$ independent on $f$ if and only if

$$
\mathscr{A}=\mathscr{A}_{p, q}:=\sup _{t>0}\left(\frac{U(t)}{V(t)}\right)^{1 / q}\left(\int_{0}^{t} V(x) \frac{d x}{x}\right)^{1 / q-1 / p}<\infty .
$$


Moreover,

$$
C=\mathscr{A}, \quad \text { if } \quad p=q
$$

and

$$
2^{-1 / p} \mathscr{A} \leq C \leq\left(\frac{p}{q}\right)^{1 / q} \mathscr{A}, \quad \text { if } \quad p<q
$$

Proof. We know from (5) that $C=I$, where

$$
I=\sup _{t>0, h \downarrow} I_{h}(t):=\sup _{t>0, h \downarrow} \frac{\left(\int_{0}^{t} d x \int_{0}^{h(x)} u(x y) d y\right)^{1 / q}}{\left(\int_{0}^{t} d x \int_{0}^{h(x)} v(x y) d y\right)^{1 / p}}
$$

and thus, by changing variables, we find that

$$
I_{h}(t)=\frac{\left(\int_{0}^{t} U(x h(x)) \frac{d x}{x}\right)^{1 / q}}{\left(\int_{0}^{t} V(x h(x)) \frac{d x}{x}\right)^{1 / p}} .
$$

We begin with the upper bound. By using (13) we obtain

$$
\int_{0}^{t} U(x h(x)) \frac{d x}{x} \leq \mathscr{A}^{q} \int_{0}^{t}\left(\int_{0}^{x h(x)} V(s) \frac{d s}{s}\right)^{q / p-1} V(x h(x)) \frac{d x}{x}
$$

[changing the variables: $s=h(x) \xi]$

$$
=\mathscr{A}^{q} \int_{0}^{t}\left(\int_{0}^{x} V(\xi h(x)) \frac{d \xi}{\xi}\right)^{q / p-1} V(x h(x)) \frac{d x}{x}
$$

$[h(x) \leq h(\xi)$ if $\xi \in(0, x)]$

$$
\begin{aligned}
& \leq \mathscr{A}^{q} \int_{0}^{t}\left(\int_{0}^{x} V(\xi h(\xi)) \frac{d \xi}{\xi}\right)^{q / p-1} V(x h(x)) \frac{d x}{x} \\
& =\frac{p}{q} \mathscr{A}^{q}\left(\int_{0}^{t} V(\xi h(\xi)) \frac{d \xi}{\xi}\right)^{q / p} .
\end{aligned}
$$

This implies that

$$
I_{h}(t) \leq\left(\frac{p}{q}\right)^{1 / q} \mathscr{A}
$$


for all $t>0$ and $h \downarrow$. Thus, (16) brings the upper bound (15) and, in particular, $C \leq \mathscr{A}$ when $p=q$.

For the lower bound let $0<\delta<t<\infty$ and $h_{\delta}(s)$ be defined as follows

$$
h_{\delta}(s)= \begin{cases}1 & \text { if } 0 \leq s<\delta \\ \frac{\delta}{s} & \text { if } \delta \leq s<t \\ 0 & \text { if } s \geq t\end{cases}
$$

Then, by using (17), we find in the case $p<q$ that

$$
I_{\delta}^{q}(t):=I_{h_{\delta}}^{q}(t)=\frac{\int_{0}^{\delta} U(x) \frac{d x}{x}+U(\delta) \log \frac{t}{\delta}}{\left(\int_{0}^{\delta} V(x) \frac{d x}{x}+V(\delta) \log \frac{t}{\delta}\right)^{q / p}} .
$$

Since $\log \frac{t}{\delta}$ takes all the values of $(0, \infty)$, when $t>\delta$, we can choose such a $t_{\delta}$ so that

$$
\log \frac{t_{\delta}}{\delta}=\frac{1}{V(\delta)} \int_{0}^{\delta} V(x) \frac{d x}{x} .
$$

With this $t_{\delta}(18)$ gives

$$
I_{\delta}^{q}\left(t_{\delta}\right)=\frac{\int_{0}^{\delta} U(x) \frac{d x}{x}+\frac{U(\delta)}{V(\delta)} \int_{0}^{\delta} V(x) \frac{d x}{x}}{2^{q / p}\left(\int_{0}^{\delta} V(x) \frac{d x}{x}\right)^{q / p}} \geq 2^{-q / p} \frac{U(\delta)}{V(\delta)}\left(\int_{0}^{\delta} V(x) \frac{d x}{x}\right)^{1-q / p}
$$

Since $\delta>0$ is arbitrary this implies that

$$
C \geq 2^{-1 / p} \mathscr{A}, \quad p<q .
$$

In the case $p=q$ we find from (18), that

$$
I_{\delta}^{p}(t)=\frac{\int_{0}^{\delta} U(x) \frac{d x}{x}+U(\delta) \log \frac{t}{\delta}}{\int_{0}^{\delta} V(x) \frac{d x}{x}+V(\delta) \log \frac{t}{\delta}}
$$

and observe that the right hand side tends to $U(\delta) / V(\delta)$, when $t \rightarrow \infty$, so that

$$
C \geq \mathscr{A}, \quad p=q
$$

and the proof is finished. 
Now, let $\mathscr{I}$ denote the constant given by (7) when $N=2$ and $u(x, y)=$ $u(x y), v(x, y)=v(x y)$. Thus,

$$
\mathscr{I}:=\sup _{0<a, b<\infty} \frac{\left(\int_{0}^{a} \int_{0}^{b} u(x y) d x d y\right)^{1 / q}}{\left(\int_{0}^{a} \int_{0}^{b} v(x y) d x d y\right)^{1 / p}} .
$$

Moreover by using (17) with $h(x) \equiv b$ and changing variable we obtain

$$
\mathscr{I}:=\sup _{t>0} \frac{\left(\int_{0}^{t} U(x) \frac{d x}{x}\right)^{1 / q}}{\left(\int_{0}^{t} V(x) \frac{d x}{x}\right)^{1 / p}} .
$$

Obviously, Theorem 3.1 yields

$$
\mathscr{I} \leq I \leq(p / q)^{1 / q} \mathscr{A}
$$

and since $I$ and $\mathscr{A}$ are comparable because of (14) and (15) the question is whether there exists a constant $c>0$ independent on $u$ and $v$ such that

$$
\mathscr{A} \leq c \mathscr{I} .
$$

Applying the l'Hôspital test we note, that

$$
\lim _{t \rightarrow 0} \frac{\int_{0}^{t} U(x) \frac{d x}{x}}{\left(\int_{0}^{t} V(x) \frac{d x}{x}\right)^{q / p}}=\frac{p}{q} \lim _{t \rightarrow 0} \frac{U(t)}{V(t)}\left(\int_{0}^{t} V(x) \frac{d x}{x}\right)^{1-q / p}
$$

and a similar equality is valid for the limits at infinity. Since the functions involved are continuous, we conclude, that $\mathscr{A}$ and $\mathscr{I}$ are comparable in a sense, that if $\mathscr{I}<\infty$, then $\mathscr{A}<\infty$. However, the estimate (19) is no longer uniform, which can be seen from the following example:

Example 3.2. Let $0<\varepsilon<1$ and let $V_{\varepsilon}(t)$ and $U_{0}(t)$ be defined by

$$
U_{0}(t)=t \quad \text { if } \quad 0<t<\infty
$$

and

$$
V_{\varepsilon}(t)= \begin{cases}t^{\varepsilon} & \text { if } 0<t \leq 1 \\ t^{1 / \varepsilon} & \text { if } t>1\end{cases}
$$

Then

$$
\mathscr{A}_{p, p}^{p}=\sup _{t>0} \frac{U_{0}(t)}{V_{\varepsilon}(t)}=1 \text {. }
$$


We have

$$
\int_{0}^{t} U_{0}(x) \frac{d x}{x}=t, \quad t>0
$$

and

$$
\int_{0}^{t} V_{\varepsilon}(x) \frac{d x}{x}= \begin{cases}\frac{1}{\varepsilon} t^{\varepsilon} & \text { if } 0<t \leq 1, \\ \frac{1}{\varepsilon}+\varepsilon\left(t^{1 / \varepsilon}-1\right) & \text { if } t>1 .\end{cases}
$$

Thus,

$$
\mathscr{I}_{\varepsilon}^{p}(t):=\frac{\int_{0}^{t} U_{0}(x) \frac{d x}{x}}{\int_{0}^{t} V_{\varepsilon}(x) \frac{d x}{x}}= \begin{cases}\varepsilon t^{1-\varepsilon} & \text { if } 0<t \leq 1 \\ \frac{t}{\frac{1}{\varepsilon}+\varepsilon\left(t^{1 / \varepsilon}-1\right)} & \text { if } t>1\end{cases}
$$

and

$$
\mathscr{I}_{\varepsilon}^{p}:=\sup _{t>0} \mathscr{I}_{\varepsilon}^{p}(t)=\frac{\varepsilon}{1+\varepsilon}\left(\frac{1+\varepsilon}{\varepsilon}\right)^{\varepsilon} \rightarrow 0, \quad \text { when } \quad \varepsilon \rightarrow 0 .
$$

Consequently, there exists no constant $c>0$, independent on $u$ and $v$ such that, in general, the inequality (19) is true, i.e., so that $\mathscr{A} \leq c \mathscr{I}$. In particular, this means that the constants $A_{N}^{(1)}$ and $A_{N}$ from the introduction are not equivalent in general.

\section{The case $0<q<p<\infty$}

Throughout this section we let $h(x) \geq 0, h \neq 0$ a.e., denote a decreasing function on $\mathrm{R}_{+}^{N}$ and $t>0$ and use the following notations:

$$
D_{h, t}:=\left\{x \in \mathrm{R}_{+}^{N} ; h(x)>t\right\}
$$

and for an increasing sequence $\left\{t_{k}\right\} \subset \mathrm{R}_{+}$we set

$$
D_{k}=D_{h, k}:=\left\{x \in \mathrm{R}_{+}^{N} ; h(x)>t_{k}\right\}, k \in \mathrm{Z} .
$$

Obviously, $D_{k} \supset D_{k+1}$ and we define

$$
\Delta_{k}=\Delta_{h, k}:=D_{k} \backslash D_{k+1}
$$

Hence, $\Delta_{k} \bigcap \Delta_{n}=\emptyset, k \neq n$ and $\mathrm{R}_{+}^{N}=\bigcup_{k} \Delta_{k}$.

Let $0<q<p<\infty$ and $r \in \mathrm{R}_{+}$be determined from the equation $1 / r=1 / q-1 / p$. 
If $u(x) \geq 0$ and $v(x) \geq 0$ are measurable functions on $\mathrm{R}_{+}^{N}$ we define the following quantities:

$$
B^{r}:=\sup _{0 \leq h \downarrow} \int_{0}^{\infty}\left(\int_{D_{h, t}} v\right)^{-r / p} d\left(-\left(\int_{D_{h, t}} u\right)^{r / q}\right),
$$

and

$$
\mathscr{B}^{r}:=\sup _{0 \leq h \downarrow\left\{t_{k}\right\} \uparrow} \sum_{k}\left(\int_{\Delta_{k}} u\right)^{r / q}\left(\int_{D_{k}} v\right)^{-r / p} .
$$

THEOREM 4.1. Let $0<q<p<\infty$.

(i) The inequality (1) is valid for all decreasing functions with a finite constant $C>0$ independent of $f$ if and only if $\mathscr{B}<\infty$. Moreover,

$$
\mathscr{B} \leq C \leq 4^{1 / q} \mathscr{B} .
$$

(ii) The following inequality is true:

$$
\mathscr{B} \leq B \leq 2^{1 / q}\left(2^{r / q}+2^{r / p}\right)^{1 / r} \mathscr{B} .
$$

(iii) The following representation takes place:

$$
B^{r}:=\frac{\left(\int_{\mathrm{R}_{+}^{N}} u\right)^{r / q}}{\left(\int_{\mathrm{R}_{+}^{N}} v\right)^{r / p}}+\sup _{0 \leq h \downarrow} \int_{0}^{\infty}\left(\int_{D_{h, t}} u\right)^{r / q} d\left(\left(\int_{D_{h, t}} v\right)^{-r / p}\right) .
$$

Proof. For a fixed $0 \leq h \downarrow$ and an increasing sequence $\left\{t_{k}\right\}$ we define the function $f_{h}(x)$ by

$$
f_{h}(x)=\sum_{k}\left(\sum_{n \leq k}\left(\int_{\Delta_{n}} u\right)^{r / q}\left(\int_{D_{n}} v\right)^{-r / q}\right)^{1 / p} \chi_{\Delta_{k}}(x) .
$$

Then $f_{h}(x) \geq 0$ is a decreasing function and

$$
\int_{\mathrm{R}_{+}^{N}} f_{h}^{p} v=\sum_{k}\left(\sum_{n \leq k}\left(\int_{\Delta_{n}} u\right)^{r / q}\left(\int_{D_{n}} v\right)^{-r / q}\right) \int_{\Delta_{k}} v
$$

[changing the order of sums]

$$
=\sum_{n}\left(\int_{\Delta_{n}} u\right)^{r / q}\left(\int_{D_{n}} v\right)^{-r / q} \sum_{k \geq n} \int_{\Delta_{k}} v
$$


[using $\sum_{k \geq n} \int_{\Delta_{k}} v=\int_{D_{n}} v,-r / q+1=-r / p$ ]

$$
=\sum_{n}\left(\int_{\Delta_{n}} u\right)^{r / q}\left(\int_{D_{n}} v\right)^{-r / p}:=\mathscr{B}_{h,\left\{t_{k}\right\}}^{r} .
$$

Suppose now that (1) is valid with a finite constant $C>0$, and assume temporarily that $\mathscr{B} \in(0, \infty)$. Then, for any $h \downarrow$ and $\left\{t_{k}\right\}$ such that $\mathscr{B}_{h,\left\{t_{k}\right\}}^{r}>0$, we obtain by using the representation formula (27),

$$
\begin{aligned}
C^{q}\left(\mathscr{B}_{h,\left\{t_{k}\right\}}\right)^{q r / p} & =C^{q}\left(\int_{\mathrm{R}_{+}^{N}} f_{h}^{p} v\right)^{q / p} \geq \int_{\mathrm{R}_{+}^{N}} f_{h}^{q} u \\
& =\sum_{k} \int_{\Delta_{k}} u\left(\sum_{n \leq k}\left(\int_{\Delta_{n}} u\right)^{r / q}\left(\int_{D_{n}} v\right)^{-r / q}\right)^{q / p}
\end{aligned}
$$

[reducing the interior sum to one term with $k=n$ ]

$$
\geq \sum_{k}\left(\int_{\Delta_{k}} u\right)^{r / q}\left(\int_{D_{n}} v\right)^{-r / p}=\mathscr{B}_{h,\left\{t_{k}\right\}}^{r} .
$$

Hence,

$$
C \geq \mathscr{B}_{h,\left\{t_{k}\right\}}
$$

and the lower bound (24) follows. The temporary assumption $\mathscr{B} \in(0, \infty)$ can be removed in the usual way (see [4], p. 178).

Next we consider the upper bound. Given $f \downarrow$ we define

$$
U(t)=\int_{D_{f, t}} u ; \quad V(t)=\int_{D_{f, t}} v .
$$

Obviously, $U(t)$ and $V(t)$ are decreasing functions.

Now we construct a special increasing sequence $\left\{\tau_{k}\right\} \subset R_{+}$as follows: Put

$$
\begin{array}{rlrl}
\tau_{0} & =1, \\
\tau_{k+1} & =\inf \left\{t: \min \left(\frac{V\left(\tau_{k}\right)}{V(t)}, \frac{U\left(\tau_{k}\right)}{U(t)}\right)=2\right\}, & & k \geq 0, \\
\tau_{k-1} & =\sup \left\{t: \min \left(\frac{V(t)}{V\left(\tau_{k}\right)}, \frac{U(t)}{U\left(\tau_{k}\right)}\right)=2\right\}, & & k \leq 0,
\end{array}
$$


and let

$$
\begin{aligned}
& Z_{1}=\left\{k \in Z: V\left(\tau_{k+1}\right)=\frac{1}{2} V\left(\tau_{k}\right)\right\}, \\
& Z_{2}=\left\{k \in Z: U\left(\tau_{k+1}\right)=\frac{1}{2} U\left(\tau_{k}\right)\right\} .
\end{aligned}
$$

We assume without a loss of generality that

$$
\mathrm{z}=\mathrm{z}_{1} \bigcup \mathrm{z}_{2}
$$

and note that $Z_{1} \cap Z_{2}=\emptyset$. Now, we write

$$
I:=\int_{\mathrm{R}_{+}^{N}} f^{q} u=\sum_{k} \int_{\Delta_{k-1}} f^{q} u
$$

where

$$
\Delta_{k}:=\Delta_{f, k}=D_{f, k} \backslash D_{f, k+1}:=D_{k} \backslash D_{k+1} .
$$

Since

$$
\tau_{k}<f(x) \leq \tau_{k+1}, \quad x \in \Delta_{k},
$$

we find

$$
\begin{aligned}
I \leq \sum_{k} \tau_{k}^{q} \int_{\Delta_{k-1}} u=\sum_{k} \frac{\tau_{k}^{q}\left(\int_{\Delta_{k-1}} u\right)^{q / p}}{\left(\sum_{n \leq k}\left(\int_{\Delta_{n-1}} u\right)^{r / p} V^{-r / p}\left(\tau_{n}\right)\right)^{q / r}} \\
\quad \cdot\left(\int_{\Delta_{k-1}} u\right)^{1-q / p}\left(\sum_{n \leq k}\left(\int_{\Delta_{n-1}} u\right)^{r / p} V^{-r / p}\left(\tau_{n}\right)\right)^{q / r}
\end{aligned}
$$

[applying Hölder's inequality with $\frac{p}{q}$ and $\frac{r}{q}$ ]

$$
\begin{aligned}
& \leq\left(\sum_{k} \frac{\tau_{k}^{p} \int_{\Delta_{k-1}} u}{\left(\sum_{n \leq k}\left(\int_{\Delta_{n-1}} u\right)^{r / p} V^{-r / p}\left(\tau_{n}\right)\right)^{p / r}}\right)^{q / p} \\
& \cdot\left(\sum_{k}\left(\int_{\Delta_{k-1}} u\right) \sum_{n \leq k}\left(\int_{\Delta_{k-1}} u\right)^{r / q} V^{-r / p}\left(\tau_{n}\right)\right)^{q / r}:=I_{1}^{q / p} I_{2}^{q / r} .
\end{aligned}
$$

We have

$$
\sum_{n \leq k}\left(\int_{\Delta_{n-1}} u\right)^{r / p} V^{-r / p}\left(\tau_{n}\right) \geq\left(\int_{\Delta_{k-1}} u\right)^{r / p} V^{-r / p}\left(\tau_{n}\right) .
$$


Thus,

$$
I_{1} \leq \sum_{k} \tau_{k}^{p} V\left(\tau_{k}\right)
$$

We also note that the sequence $\left\{\tau_{k}\right\}$ is constructed in such a way that

$$
V\left(\tau_{k}\right) \geq 2 V\left(\tau_{k+1}\right), \quad U\left(\tau_{k}\right) \geq 2 U\left(\tau_{k+1}\right) \quad \text { for all } \quad k \in \mathrm{Z}
$$

Therefore, in particular,

$$
V\left(\tau_{k}\right)=\int_{\Delta_{k}} v+V\left(\tau_{k+1}\right) \geq 2 V\left(\tau_{k+1}\right) .
$$

Hence,

$$
V\left(\tau_{k+1}\right) \leq \int_{\Delta_{k}} v
$$

and, consequently,

$$
V\left(\tau_{k}\right) \leq 2 \int_{\Delta_{k}} v
$$

This implies that

$$
I_{1} \leq 2 \sum_{k} \tau_{k}^{p} \int_{\Delta_{k}} v \leq 2 \sum_{k} \int_{\Delta_{k}} f^{p} v \leq 2 \int_{\mathrm{R}_{+}^{N}} f^{p} v .
$$

Now we return to the estimate of $I_{2}$. Write

$$
\begin{aligned}
I_{2} & =\sum_{n}\left(\int_{\Delta_{n-1}} u\right)^{r / p} V^{-r / p}\left(\tau_{n}\right) \sum_{k \geq n} \int_{\Delta_{k-1}} u \\
& =\sum_{n}\left(\int_{\Delta_{n-1}} u\right)^{r / p} V^{-r / p}\left(\tau_{n}\right) U\left(\tau_{n-1}\right):=I_{2,1}+I_{2,2},
\end{aligned}
$$

where, using (29), we put

$$
I_{2,1}=\sum_{n: n-1 \in \mathrm{Z}_{1}}\left(\int_{\Delta_{n-1}} u\right)^{r / p} V^{-r / p}\left(\tau_{n}\right) U\left(\tau_{n-1}\right),
$$

and

$$
I_{2,2}=\sum_{n: n-1 \in \mathrm{Z}_{2}}\left(\int_{\Delta_{n-1}} u\right)^{r / p} V^{-r / p}\left(\tau_{n}\right) U\left(\tau_{n-1}\right) .
$$


Similar to the derivation of (31) we obtain that

$$
U\left(\tau_{k}\right) \leq 2 \int_{\Delta_{k}} u
$$

Hence, by using (28) and (32), we find that

$$
\begin{aligned}
I_{2,1} \leq 2 \sum_{n: n-1 \in \mathrm{Z}_{1}}\left(\int_{\Delta_{n-1}} u\right)^{r / q}\left(\frac{1}{2} V\left(\tau_{n-1}\right)\right)^{-r / p} & \\
& \leq 2^{1+r / p} \sum_{n}\left(\int_{\Delta_{n}} u\right)^{r / q} V^{-r / p}\left(\tau_{n}\right) \leq 2^{1+r / p} \mathscr{B}^{r} .
\end{aligned}
$$

For the second term we use again (28) and (32). We have

$$
\begin{gathered}
U\left(\tau_{n-1}\right)=2 U\left(\tau_{n}\right), \quad n-1 \in \mathrm{Z}_{2}, \\
\int_{\Delta_{n-1}} u=U\left(\tau_{n-1}\right)-U\left(\tau_{n}\right)=U\left(\tau_{n}\right) \leq 2 \int_{\Delta_{n}} u .
\end{gathered}
$$

Thus,

$$
I_{2,2} \leq 2^{1+r / p} \sum_{n: n-1 \in Z_{2}}\left(\int_{\Delta_{n}} u\right)^{r / q} V^{-r / p}\left(\tau_{n}\right) \leq 2^{1+r / p} \mathscr{B}^{r} .
$$

Summarizing the above estimates we obtain the upper bound

$$
\left(\int_{\mathrm{R}_{+}^{N}} f^{q} u\right)^{1 / q} \leq 4^{1 / q} \mathscr{B}\left(\int_{\mathrm{R}_{+}^{N}} f^{p} v\right)^{1 / p}
$$

and the part (i) of the Theorem 4.1 is proved.

For the proof of the lower bound (25) we fix $0 \leq h(x) \downarrow$ and define

$$
\Delta_{k, t}=\left\{x: t<h(x) \leq t_{k+1}\right\} .
$$

Then

$$
\left(\int_{\Delta_{k}} u\right)^{r / q}=\int_{t_{k}}^{t_{k+1}} d\left(-\left(\int_{\Delta_{k, t}} u\right)^{r / q}\right)=\frac{r}{q} \int_{t_{k}}^{t_{k+1}}\left(\int_{\Delta_{k, t}} u\right)^{r / p} d\left(-\int_{\Delta_{k, t}} u\right) .
$$

Since

$$
\int_{\Delta_{k, t}} u \leq U(t) ; d\left(-\int_{\Delta_{k, t}} u\right)=d\left(-U(t)+\int_{D_{k+1}} u\right)=d(-U(t))
$$


we obtain

$$
\left(\int_{\Delta_{k}} u\right)^{r / q} \leq \frac{r}{q} \int_{t_{k}}^{t_{k+1}} U^{r / p}(t) d(-U(t))=\int_{t_{k}}^{t_{k+1}} d\left(-U^{r / q}(t)\right) .
$$

Applying this estimate and that

$$
\left(\int_{D_{k}} v\right)^{-r / p}=V^{-r / p}\left(t_{k}\right) \leq V^{-r / p}(t), \quad t \in\left[t_{k}, t_{k+1}\right],
$$

we find

$$
\begin{aligned}
\sum_{k}\left(\int_{\Delta_{k}} u\right)^{r / q}\left(\int_{D_{k}} v\right)^{-r / p} & \leq \sum_{k} \int_{t_{k}}^{t_{k+1}} V^{-r / p}(t) d\left(-U^{r / q}(t)\right) \\
& \leq \int_{0}^{\infty} V^{-r / p}(t) d\left(-U^{r / q}(t)\right) \leq B^{r}
\end{aligned}
$$

Thus,

$$
\mathscr{B} \leq B
$$

For the proof of the upper bound (25) we observe that for $0 \leq h(x) \downarrow$ and an increasing sequence $\left\{t_{k}\right\} \subset \mathrm{R}_{+}$we have

$$
\begin{aligned}
B_{h}^{r}:=\int_{0}^{\infty} V^{-r / p}(t) d\left(-U^{r / q}(t)\right) & =\sum_{k} \int_{t_{k}}^{t_{k+1}} V^{-r / p}(t) d\left(-U^{r / q}(t)\right) \\
& \leq \sum_{k} V^{-r / p}\left(t_{k+1}\right) U^{r / q}\left(t_{k}\right):=\mathscr{I} .
\end{aligned}
$$

Now suppose that $\left\{t_{k}\right\}$ is taken in the same way as the sequence $\left\{\tau_{k}\right\}$ was taken in the proof of part (i), that is $t_{k}=\tau_{k}, k \in \mathrm{Z}$. Then

$$
\mathscr{I}=\sum_{k \in \mathrm{Z}_{1}}+\sum_{k \in \mathrm{Z}_{2}}:=\mathscr{I}_{1}+\mathscr{I}_{2}
$$

Therefore, by using (30), (31) and (32), we find that

$$
\begin{aligned}
& \mathscr{I}_{1} \leq 2^{r / q+r / p} \sum_{k \in Z_{1}} V^{-r / p}\left(\tau_{k}\right)\left(\int_{\Delta_{k}} u\right)^{r / q}, \\
& \mathscr{I}_{2} \leq 2^{2 r / q} \sum_{k \in Z_{2}} V^{-r / p}\left(\tau_{k+1}\right)\left(\int_{\Delta_{k+1}} u\right)^{r / q} .
\end{aligned}
$$


Thus,

$$
\mathscr{I} \leq 2^{r / q}\left(2^{r / q+r / p}\right) \sum_{k \in Z} V^{-r / p}\left(\tau_{k}\right)\left(\int_{\Delta_{k}} u\right)^{r / q} \leq 2^{r / q}\left(2^{r / q+r / p}\right) \mathscr{B}^{r} .
$$

This implies that

$$
B \leq 2^{1 / q}\left(2^{r / q+r / p}\right)^{1 / r} \mathscr{B}
$$

and, hence, the upper bound (25) is proved.

For the proof of part (iii) we suppose first that $B<\infty$. Then by putting, for a fixed $0 \leq h(x) \downarrow$,

$$
V(t)=\int_{D_{h, t}} v, \quad U(t)=\int_{D_{h, t}} u,
$$

we see that

$$
\infty>B^{r} \geq \int_{\tau}^{\infty} V^{-r / q}(t) d\left(-U^{r / q}(t)\right) \rightarrow 0, \quad \tau \rightarrow \infty .
$$

Hence,

$$
\int_{\tau}^{\infty} V^{-r / p}(t) d\left(-U^{r / q}(t)\right) \geq V^{-r / q}(\tau) U^{r / q}(\tau) \rightarrow 0, \quad \tau \rightarrow \infty .
$$

This implies, by integration by parts, that

$$
\int_{0}^{\infty} V^{-r / p}(t) d\left(-U^{r / q}(t)\right)=\frac{U^{r / q}(0)}{V^{r / p}(0)}+\int_{0}^{\infty} U^{r / q}(t) d V^{-r / p}(t)
$$

and the inequality

$$
\infty>B^{r} \geq \frac{\left(\int_{\mathrm{R}_{+}^{N}} u\right)^{r / q}}{\left(\int_{\mathrm{R}_{+}^{N}} v\right)^{r / p}}+\sup _{0 \leq h} \int_{0}^{\infty}\left(\int_{D_{h, t}} u\right)^{r / q} d\left(\int_{D_{h, t}} v\right)^{-r / p}
$$

follows.

Now suppose that the right hand side of (26) is finite. Then, for a fixed $h \downarrow$, integration by parts gives

$$
\int_{0}^{\infty} U^{r / q}(t) d V^{-r / p}(t) \geq-\frac{U^{r / q}(0)}{V^{r / p}(0)}+\int_{0}^{\infty} V^{-r / p}(t) d\left(-U^{r / q}(t)\right)
$$

and we obtain the reversed inequality to (33). Thus, also (26) is proved and the proof is complete.

$$
\text { EXAMPLE 4.2. Let } v=u \in L^{1}\left(\mathrm{R}_{+}^{N}\right) \text {. Then } B^{r}=\frac{r}{q} \int_{\mathrm{R}_{+}^{N}} v \text {. }
$$




\section{REFERENCES}

1. Barza, S., Persson L. E. and Soria, J., Sharp weighted multidimensional integral inequalities for monotone functions, Math. Nachr. 210 (2000), 43-58.

2. Carro, M. J., Pick, L., Soria, J. and Stepanov, V., On embeddings between clasical Lorentz spaces, Math. Ineq. Appl., to appear.

3. Krasnosel'ski, M. A. and Ruticki, Ya. B., Convex Functions and Orlicz spaces, Noordhoff, Groningen, 1961.

4. Stepanov V., The weighted Hardy's inequality for nonincreasing functions, Trans. Amer. Math. Soc. 338 (1993), 173-186.

DEPARTMENT OF MATHEMATICS

LULEÅ UNIVERSITY

S-97187 LULEÅ

SWEDEN

E-mail: sorina@sm.luth.se

E-mail:1arserik@sm.luth.se
COMPUTER CENTER OFFAR-EASTERN BRANCH OF THE RUSSIAN ACADEMY OF SCIENCE SHELEST 118-205, KHABAROVSK 680042 RUSSIA

E-mail: stepanov@as.fe.ru 